\title{
The Bergman Kernel on Monomial Polyhedra
}

\author{
CHIEH-HSIEN TIAO
}

\section{Introduction}

In order to understand the Bergman kernel for a complex domain $\Omega$ in $\mathbb{C}^{n}$ at $z$ close to the boundary $\partial \Omega$, we usually insert the biholomorphic image of a polydisc $\mathcal{D}$ centered at $z$ in $\Omega$ to generate the upper bound for the Bergman kernel on $\Omega$ :

$$
K_{\Omega}(z, z) \leq K_{\mathcal{D}}(z, z)=\frac{1}{\operatorname{Vol}(\mathcal{D})} .
$$

On the other hand, Catlin [3] showed by using a $\bar{\partial}$ estimate that, on a finite type pseudoconvex domain $\Omega$ in $\mathbb{C}^{2}$, there exists a polydisc $\mathcal{D}$ such that

$$
K_{\Omega}(z, z) \geq c \cdot \frac{1}{\operatorname{Vol}(\mathcal{D})} ;
$$

the same formula was later shown by McNeal [8] on convex domains in $\mathbb{C}^{n}$. A question arises: Are polydiscs enough to describe the Bergman kernel for smooth bounded domains?

For a general domain in $\mathbb{C}^{n}$, it is not always possible to find a polydisc $D$ that models the domain. Consider $\Omega \subset \mathbb{C}^{3}$ defined by $\left|z_{1}\right|^{10}+\left|z_{2}\right|^{10}+\left|z_{1} z_{2}\right|^{2}+\left|z_{3}\right|^{2}<$ 1 , and let $z=(0,0,1-\varepsilon)$. It is easy to show that all polydiscs centered at $z$ in $\Omega$ have maximal volume of approximately $\varepsilon^{4}$; thus, the upper bound of the Bergman kernel at $z$ obtained by inserting polydiscs is roughly $\varepsilon^{-4}$. But consider a Reinhardt domain $\mathcal{R}$ centered at $z$ bounded by $\left|z_{1}\right|<1,\left|z_{2}\right|<1,\left|z_{3}-(1-\varepsilon)\right|<$ $\varepsilon / 2$, and $\left|z_{1} z_{2}\right|<\varepsilon / 2$. The volume of $\mathcal{R}$ is roughly $\varepsilon^{4}(-\log \varepsilon+1)$, which is much larger than $\varepsilon^{4}$ when $\varepsilon \ll 1$; therefore, the upper bound at $z$ given by $\mathcal{R}$ is $1 / \varepsilon^{4}(-\log \varepsilon+1)$, much smaller than the ones given by any polydiscs.

The preceding example shows that polydiscs do not provide a good enough way of estimating upper bounds for the Bergman kernel. Instead of trying to fit a poly$\operatorname{disc} \mathcal{D}$ about the point $z$ into $\Omega$, it seems better to try to fit the largest "monomial polyhedron" $P$ about $z$ into $\Omega$, where a monomial polyhedron $P$ associated with a finite subcollection $\mathcal{B}$ of index space $\mathcal{N}^{n}, \mathcal{N}=\mathbb{N} \cup\{0\}$, is defined as follows.

Definition 1.1. A domain $P$ in $\mathbb{C}^{n}$ is a monomial polyhedron if there exists a subset $\mathcal{B}=\left\{\alpha_{1}, \ldots, \alpha_{m}\right\}$ of $\mathcal{N}^{n}$ and, for each $\alpha \in \mathcal{B}$, there exists a unique $C_{\alpha} \in \mathbb{R}$ such that $P=P(\mathcal{B})=\left\{z \in \mathbb{C}^{n}:\left|z^{\alpha}\right|<e^{C_{\alpha}}, \alpha \in \mathcal{B}\right\}$.

Received October 23, 1997. Revision received July 22, 1998.

Michigan Math. J. 46 (1999). 
Note that $\mathcal{R}$ in the foregoing example is a monomial polyhedron. Also, it is obvious that the $\log$ domain of $P$ defined as

$$
\log (P)=\left\{w \in \mathbb{R}^{n}: \alpha \cdot w<c_{\alpha}=\log d_{\alpha}, \alpha \in \mathcal{B}\right\}
$$

is an unbounded polyhedron containing $(-\infty, \ldots,-\infty)$.

It is possible that, among the inequalities in (1) that define $\log (P)$, some may be redundant. However we will show that we can assume $\mathcal{B}$ satisfies: (i) $\mathcal{B}$ is a minimal collection defining $P$; and (ii) for $\alpha=\left(\alpha_{1}, \ldots, \alpha_{n}\right) \in \mathcal{B}, \alpha_{1}, \ldots, \alpha_{n}$ are relatively prime. Such a set $\mathcal{B}$ is unique with respect to $P$. We call such a set $\mathcal{B}$ a regular index set for $P$.

In order to precisely define our estimate of the Bergman kernel, we must first define the representation of the faces of the convex monomial polyhedron $\log (P)$. For each $\mathcal{A} \subseteq \mathcal{B}$, define a face $\mathcal{F}=\mathcal{F}(\mathcal{A})$ of $\partial \log (P)$ determined by $\mathcal{A}$ by

$$
\mathcal{F}(\mathcal{A})=\left\{w \in \overline{\log (P)}: \alpha \cdot w=c_{\alpha} \forall \alpha \in \mathcal{A} \text { and } \alpha \cdot w<c_{\alpha} \forall \alpha \in \mathcal{B}-\mathcal{A}\right\} .
$$

Of course, there is no guarantee that such a face $\mathcal{F}$ is not empty. However, if it is not empty then we can conversely determine a subcollection $\mathcal{A}=\mathcal{A}(\mathcal{F})$ of $\mathcal{B}$ by $\mathcal{A}(\mathcal{F})=\left\{\alpha \in \mathcal{B}: \alpha \cdot w=c_{\alpha} \forall w \in \mathcal{F}\right\}$, and we will show (in Proposition 1.8) that it is a one-to-one correspondence map between non-empty faces and the subcollections that determine non-empty faces.

We will see later that, in order to estimate the Bergman kernel, we need only study the bounded faces of $\log (P)$. Thus we will define $\mathbb{F}=\{\mathcal{F}: \mathcal{F}$ is bounded $\}$ and $\mathcal{U}(\mathcal{F})=\left\{\beta \in \mathcal{N}^{n}: \beta=\sum_{\alpha \in \mathcal{A}(\mathcal{F})} \lambda_{\alpha} \alpha, 0<\lambda_{\alpha} \leq 1\right\}$. Notice that if we let $|\mathcal{K}|$ be the cardinality of $\mathcal{K}$, then both $|\mathbb{F}|$ and $|\mathcal{U}(\mathcal{F})|$ are finite.

Define $P$ as $(M, \varepsilon)$-nondegenerate for some $M$ and $\varepsilon>0$ if: (i) $\sum_{j=1}^{n} \alpha_{j} \leq$ $M$ for all $\alpha=\left(\alpha_{1}, \ldots, \alpha_{n}\right) \in \mathcal{B}$; (ii) $\mathcal{A}(\mathcal{F})$ is a linearly independent set for all $\mathcal{F} \in \mathcal{B}$; and (iii) every $\mathcal{F} \in \mathcal{B}$ contains an $\varepsilon$-ball in the corresponding dimension. We will give a more precise description in Definition 1.10. Now let us state our theorem.

TheORem 3.1. Let $P$ be an $(M, \varepsilon)$-nondegenerate bounded monomial polyhedron, and let $\zeta_{\beta}(z)=z^{\beta}$. Then there are constants $C>c>0$ depending on $M$, $\varepsilon$, and $n$ such that the Bergman kernel for $P$ can be estimated as

$$
c \cdot K_{P}(z, z)<\sum_{\mathcal{F} \in \mathbb{F}}\left(\prod_{\alpha \in \mathcal{A}(\mathcal{F})} \frac{1}{\left(1-\left|\left(z^{\alpha} / d_{\alpha}\right)\right|^{2}\right)^{2}} \cdot \sum_{\beta+\overline{\mathbf{1}} \in \mathcal{U}(\mathcal{F})} \frac{\left|z^{\beta}\right|^{2}}{\left\|\zeta_{\beta}\right\|^{2}}\right)<C \cdot K_{P}(z, z) \text {. }
$$

Furthermore, for $\beta+\overline{\mathbf{1}} \in \mathcal{U}(\mathcal{F})$ and with constants $C>c>0$ depending on $\varepsilon, M$, and $n$, we have

$$
c \cdot\left\|z^{\beta}\right\|^{2}<\left|\tilde{z}^{\beta+\overline{\mathbf{1}}}\right|^{2} \cdot A^{n-k}(\mathcal{F})<C \cdot\left\|z^{\beta}\right\|^{2},
$$

where $\tilde{z}=e^{\tilde{w}}$ for any $\tilde{w} \in \mathcal{F}$ and $A^{n-k}(\mathcal{F})$ is the volume of $\mathcal{F}$ in its corresponding dimension, which will be described more precisely in Sections 2 and 3.

REMARK 3.1. $\left|\tilde{z}^{\beta+\overline{\mathbf{1}}_{\mid}}\right|$is independent of the choice of $\tilde{w}$ as long as $\beta+\overline{\mathbf{1}} \in \mathcal{U}(\mathcal{F})$ and $\tilde{w} \in \mathcal{F}$. 
This paper is part of my thesis. The problem was provided by my thesis advisor, David Catlin. During the study, he always inspired me with valuable ideas and suggestions. Therefore I would like to express my sincere gratitude to him.

\section{Geometry of Bounded Monomial Polyhedrons}

In order to describe the Bergman kernel $K(z, w)$ for a Reinhardt domain by utilizing the formula, with $\zeta_{\beta}(z)=z^{\beta}$,

$$
K(z, w)=\sum_{\beta \in \mathcal{N}^{n}} \frac{z^{\beta} \bar{w}^{\beta}}{\left\|\zeta_{\beta}\right\|^{2}},
$$

where $\mathcal{N}=\mathbb{N} \cup\{0\}$, it is desirable to estimate $\left\|z^{\beta}\right\|$ for all $\beta \in \mathcal{N}^{n}$. Thus it becomes important to better understand the geometry of the underlying domains.

In our paper, we assume $\mathcal{N}^{n}=\left\{\left(\alpha_{1}, \ldots, \alpha_{n}\right): \alpha_{j} \in \mathbb{N} \cup\{0\}\right\}$. Let $\Omega$ be a bounded Reinhardt domain and let

$$
\log (\Omega)=\left\{\left(w_{1}, \ldots, w_{n}\right) \in \mathbb{R}^{n}:\left(e^{w_{1}}, \ldots, e^{w_{n}}\right) \in \Omega\right\} .
$$

We want to give a definition for monomial polyhedrons.

DeFINITION 1.1. A domain $P$ in $\mathbb{C}^{n}$ is a monomial polyhedron if there exists a subset $\mathcal{B}=\left\{\alpha_{1}, \ldots, \alpha_{m}\right\}$ of $\mathcal{N}^{n}$ and, for each $\alpha \in \mathcal{B}$, there exists a unique $C_{\alpha} \in \mathbb{R}$ such that

$$
P=P(\mathcal{B})=\left\{z \in \mathbb{C}^{n}:\left|z^{\alpha}\right|<e^{C_{\alpha}}, \alpha \in \mathcal{B}\right\}
$$

Because a monomial polyhedron $P$ thus defined is a Reinhardt domain, we see that

$$
\log (P)=\left\{w \in \mathbb{R}^{n}: \alpha \cdot w<C_{\alpha}, \alpha \in \mathcal{B}\right\}
$$

Definition 1.2. We say $\alpha=\left(a_{1}, \ldots, a_{n}\right) \in \mathcal{N}^{n}$ is prime if $a_{1}, \ldots, a_{n}$ are relatively prime.

Without loss of generality, we can assume that all $\alpha$ in $\mathcal{B}$ are prime. Let us denote

$$
\mathcal{W}_{\alpha}=\left\{w \in \mathbb{R}^{n}: \alpha \cdot w<C_{\alpha}\right\} .
$$

Definition 1.3. We say $\alpha$ is essential in $\mathcal{B}$ if there exists a $w \in \mathbb{R}^{n}$ such that $\alpha \cdot w \geq C_{\alpha}$ but $\beta \cdot w<C_{\beta}$ for all $\beta$ in $\mathcal{B}$ and $\beta \neq \alpha$. It is equivalent to say that

$$
\left(\bigcap_{\beta \in \mathcal{B}-\{\alpha\}} \mathcal{W}_{\beta}\right) \cap \mathcal{W}_{\alpha}^{C} \neq \emptyset .
$$

Also, we say $\alpha$ is non-essential in $\mathcal{B}$ if it is not essential-that is, if for all $w \in$ $\mathbb{R}^{n}$ such that $\alpha \cdot w \geq C_{\alpha}$ there exists a $\beta \in \mathcal{B}$ such that $\beta \cdot w \geq C_{\beta}$. It is equivalent to say that

$$
\bigcap_{\beta \in \mathcal{B}-\{\alpha\}} \mathcal{W}_{\beta} \subseteq \mathcal{W}_{\alpha}
$$


Let us denote $\operatorname{ess}(\mathcal{B})=\{\alpha \in \mathcal{B}: \alpha$ is essential $\}$. First we would like to show that essential indices will remain essential even after we take off non-essential indices away from $\mathcal{B}$.

Lemma 1.4. Let $\alpha$ be non-essential in $\mathcal{B}$, and let $\mathcal{B}^{\prime}=\mathcal{B}-\{\alpha\}$. Then ess $(\mathcal{B})=$ $\operatorname{ess}\left(\mathcal{B}^{\prime}\right)$.

Proof. It is obvious from (1.3) that $\operatorname{ess}(\mathcal{B}) \subseteq \operatorname{ess}\left(\mathcal{B}^{\prime}\right)$. Suppose $\gamma$ is non-essential in $\mathcal{B}$ but essential in $\mathcal{B}^{\prime}$, and let $\tilde{P}=\bigcap_{\beta \in \mathcal{B}-\{\alpha, \gamma\}} \mathcal{W}_{\beta}$.

Since both $\alpha$ and $\gamma$ are non-essential in $\mathcal{B}$, by (1.4) we have $\tilde{P} \cap \mathcal{W}_{\alpha} \subseteq \mathcal{W}_{\gamma}$ and $\tilde{P} \cap \mathcal{W}_{\gamma} \subseteq \mathcal{W}_{\alpha}$. By intersecting with $\tilde{P}$, this means $\tilde{P} \cap \mathcal{W}_{\gamma}=\tilde{P} \cap \mathcal{W}_{\alpha}$. But the assumption that $\gamma$ is essential in $\mathcal{B}^{\prime}$ and (1.3) imply that $\tilde{P} \cap \mathcal{W}_{\gamma}^{C} \neq \varnothing$. Notice that $\mathcal{W}_{\gamma}$ and $\mathcal{W}_{\alpha}$ are both half-spaces. For any two half-spaces $\mathcal{W}_{1}$ and $\mathcal{W}_{2}$, if there exists an open neighborhood $\mathcal{U}$ such that both $\mathcal{W}_{1} \cap \mathcal{U}$ and $\mathcal{W}_{1}^{C} \cap \mathcal{U}$ are non-empty and if $\mathcal{W}_{1} \cap \mathcal{U}=\mathcal{W}_{2} \cap \mathcal{U}$, then $\mathcal{W}_{1}=\mathcal{W}_{2}$. Thus, by taking $\mathcal{U}=\tilde{P}$, we have $\mathcal{W}_{\gamma}=\mathcal{W}_{\alpha}$. Finally, since all indices in $\mathcal{B}$ are prime, $\gamma=\alpha$, a contradiction.

Now denote ess $\log (P)=\left\{w \in \mathbb{R}^{n}: \alpha \cdot w<C_{\alpha}, \alpha \in \operatorname{ess}(\mathcal{B})\right\}$. It is obvious that

$$
\log (P)=\bigcap_{\alpha \in \mathcal{B}} \mathcal{W}_{\alpha} \quad \text { and } \quad \operatorname{ess} \log (P)=\bigcap_{\alpha \in \operatorname{ess}(\mathcal{B})} \mathcal{W}_{\alpha} .
$$

We would like to show the following.

LEMma 1.5. ess $\log (P)=\log (P)$.

Proof. From (1.4) and (1.5), we see that $\log (P)=\bigcap_{\beta \in \mathcal{B}-\{\alpha\}} \mathcal{W}_{\beta}$ for any nonessential index $\alpha$ in $\mathcal{B}$. And Lemma 1.4 shows that the essential indices in $\mathcal{B}$ and $\mathcal{B}^{\prime}$ are identical, where $\mathcal{B}^{\prime}=\mathcal{B}-\{\alpha\}$. By repeated application of this procedure for (finitely many times), the result follows.

From Definition 1.3, there exists an open neighborhood $\mathcal{U}$ for each $\alpha$ in ess $(\mathcal{B})$ such that $\mathcal{U} \cap \partial \log (P) \neq \varnothing$ and $\mathcal{U} \cap \log (P)=\mathcal{U} \cap \mathcal{W}_{\alpha}$. Thus, we can think of essential indices as normal vectors to local neighborhoods of $\partial \log (P)$.

Obviously, $\mathcal{B}$ must contain such normal vectors. However, if we take only those normal vectors, Lemma 1.5 implies that we obtain a unique collection of indices, $\mathcal{B}$, which describes the monomial polyhedron $P$. We would like to give a name to such a collection.

Definition 1.6. $\mathcal{B} \subset \mathcal{N}^{n}$ is a regular index set with respect to $P$ if, for all $\alpha \in$ $\mathcal{B}, \alpha$ is prime and essential in $\mathcal{B}$.

It is easy to see that a monomial polyhedron $P$ uniquely corresponds to a regular index $\mathcal{B}$, and we will assume that $\mathcal{B}$ is regular throughout this paper.

REMARK 1.1. Since we require $P$ to be bounded, from (1.2) it is easy to see that $e_{j}=(0, \ldots, 0,1,0, \ldots, 0) \in \mathcal{B}$ for all $j=1, \ldots, n$. For if not, then 
$(0, \ldots, 0, z, 0, \ldots, 0)$ satisfies all the inequalities in (1.2) for all $z \in \mathbb{C}$. Thus $P$ would be unbounded.

Next, we would like to describe the faces of $\overline{\log (P)}$.

Definition 1.7. $\mathcal{F}$ is a face of $\overline{\log (P)}$ if there exists a subcollection $\mathcal{A}$ of $\mathcal{B}$ such that

$$
\begin{aligned}
& \mathcal{F}=\mathcal{F}(\mathcal{A})=\{w \in \overline{\log (P)}: \alpha \cdot w=C_{\alpha} \text { for all } \alpha \in \mathcal{A} \text { and } \\
&\left.\alpha \cdot w<C_{\alpha} \text { for all } \alpha \in \mathcal{B}-\mathcal{A}\right\} .
\end{aligned}
$$

It follows from (1.6) that if $\mathcal{A}_{1}$ and $\mathcal{A}_{2}$ are subcollections of $\mathcal{B}$ such that $\mathcal{A}_{1} \neq \mathcal{A}_{2}$ and one of $\mathcal{F}\left(\mathcal{A}_{1}\right)$ and $\mathcal{F}\left(\mathcal{A}_{2}\right)$ is not empty, then $\mathcal{F}\left(\mathcal{A}_{1}\right) \neq \mathcal{F}\left(\mathcal{A}_{2}\right)$. Thus, for each non-empty face $\mathcal{F}$ there corresponds an index set

$$
\mathcal{A}=\mathcal{A}(\mathcal{F})=\left\{\alpha \in \mathcal{B}: \alpha \cdot w=C_{\alpha} \text { for all } w \in \mathcal{F}\right\} .
$$

But it follows directly from the definition that

$$
\mathcal{A}\left(\mathcal{F}\left(\mathcal{A}_{0}\right)\right)=\mathcal{A}_{0} \quad \text { and } \quad \mathcal{F}\left(\mathcal{A}\left(\mathcal{F}_{0}\right)\right)=\mathcal{F}_{0}
$$

once $\mathcal{F}\left(\mathcal{A}_{0}\right)$ and $\mathcal{F}_{0}$ are not empty. Thus, by collecting all non-empty faces and corresponding index sets as

$$
\overline{\mathbb{F}}=\{\mathcal{F} \subseteq \overline{\log (P)}: \mathcal{F} \text { is a non-empty face }\}
$$

and

$$
\overline{\mathbb{A}}=\{\mathcal{A} \subseteq \mathcal{B}: \mathcal{F}(\mathcal{A}) \text { is non-empty }\},
$$

we have the following proposition.

\section{Proposition 1.8.}

(i) There exists a one-to-one onto map between $\overline{\mathbb{F}}$ and $\overline{\mathbb{A}}$;

(ii) $\overline{\log (P)}$ is a disjoint union of all $\mathcal{F}$ in $\mathbb{F}$.

We also need the following.

Lemma 1.9. For $\mathcal{F}_{1}, \mathcal{F}_{2} \in \overline{\mathbb{F}}, \overline{\mathcal{F}}_{1}=\overline{\mathcal{F}}_{2}$ if and only if $\mathcal{F}_{1}=\mathcal{F}_{2}$.

Proof. The "if" part is obvious. For the "only if" part, first notice that for each $\mathcal{F}$ there exists an $\mathcal{A}$ such that (1.6) holds. But then $\overline{\mathcal{F}}$ becomes

$$
\begin{aligned}
\overline{\mathcal{F}} & =\left\{w \in \overline{\log (P)}: \alpha \cdot w=C_{\alpha} \text { for all } \alpha \in \mathcal{A}\right. \text { and } \\
& \left.\alpha \cdot w \leq C_{\alpha} \text { for all } \alpha \in \mathcal{B}-\mathcal{A}\right\} \\
= & \bigcup_{\mathcal{A} \subseteq \mathcal{A}^{\prime}}\left\{w \in \overline{\log (P)}: \alpha \cdot w=C_{\alpha} \text { for all } \alpha \in \mathcal{A}^{\prime}\right. \text { and } \\
= & \left.\quad \alpha \cdot w<C_{\alpha} \text { for all } \alpha \in \mathcal{B}-\mathcal{A}^{\prime}\right\} \\
\bigcup_{\mathcal{A} \subseteq \mathcal{A}^{\prime}} \mathcal{F}\left(\mathcal{A}^{\prime}\right) . &
\end{aligned}
$$


By Proposition 1.8(ii), this expression is a disjoint union. It follows that, if $\mathcal{F}_{1}=$ $\mathcal{F}\left(\mathcal{A}_{1}\right)$ and $\mathcal{F}_{2}=\mathcal{F}\left(\mathcal{A}_{2}\right)$, then $\overline{\mathcal{F}}_{1}=\overline{\mathcal{F}}_{2}$ implies $\mathcal{F}_{1} \subseteq \overline{\mathcal{F}}_{2}$ and $\mathcal{F}_{2} \subseteq \overline{\mathcal{F}}_{1}$, which implies $\mathcal{A}_{1}=\mathcal{A}_{2}$, which in turn implies $\mathcal{F}_{1}=\mathcal{F}_{2}$.

To make our computation manageable, we need to impose some extra conditions on our monomial polyhedrons.

DEFINITION 1.10. A monomial polyhedron $P$ is $(M, \varepsilon)$-nondegenerate for some $M, \varepsilon>0$ if all of the following conditions hold.

(i) For all $\alpha=\left(\alpha_{1}, \ldots, \alpha_{n}\right) \in \mathcal{B}, \alpha_{1}+\cdots+\alpha_{n} \leq M$.

(ii) For all $\mathcal{A}$ in $\overline{\mathbb{A}}$, the set of elements in $\mathcal{A}$ is linearly independent in $\mathbb{R}^{n}$.

(iii) For all $\mathcal{F}$ in $\overline{\mathbb{F}}$, let $\mathbf{B}_{\varepsilon}(w)$ be an Euclidean ball centered at $w$ with radius $\varepsilon$, and let $\mathcal{W}_{\mathcal{F}}=\left\{w: \alpha \cdot w=C_{\alpha}\right.$ for all $\left.\alpha \in \mathcal{A}(\mathcal{F})\right\}$; then there exists a $w$ in $\mathcal{F}$ such that $\mathbf{B}_{\varepsilon}(w) \cap \mathcal{W}_{\mathcal{F}} \subseteq \mathcal{F}$.

Notice that, for any monomial polyhedron $P$, there exist $M, \varepsilon>0$ that satisfy conditions (i) and (iii). We emphasize the roles played by $M$ and $\varepsilon>0$ because our result will depend on both $M$ and $\varepsilon$.

In order to sum (1.1), we need to suitably decompose the index space $\mathcal{N}^{n}$. One natural way of doing so is by decomposing the index space into a finite disjoint union of convex cones generated by elements in $\overline{\mathbb{A}}$, as follows.

Let $\mathcal{A}=\left\{\alpha_{1}, \ldots, \alpha_{k}\right\} \subset \mathcal{N}^{n}$. We say $\alpha$ is a nonnegative combination of $\mathcal{A}$ if there exist $\lambda_{1}, \ldots, \lambda_{k}$ and $\lambda_{j} \geq 0$ for all $j=1, \ldots, k$ such that $\alpha=\sum_{j=1}^{k} \lambda_{j} \alpha_{j}$; we say $\alpha$ is a positive combination of $\mathcal{A}$ if $\alpha$ is a nonnegative combination of $\mathcal{A}$ as just described while also requiring $\lambda_{j}>0$ for all $j=1, \ldots, k$. We say $\Gamma(\mathcal{A})$ is the open convex cone generated by $\mathcal{A}$ if $\Gamma(\mathcal{A})=\{\alpha: \alpha$ is a positive combination of $\mathcal{A}$ \}.

Note that the term "open" used here is not in a traditional sense, for the set $\Gamma(\mathcal{A})$ is discrete. Rather, we use "open" to emphasize that this cone does not contain the boundary.

The following proposition will imply that the index space $\mathcal{N}^{n}$ is a (finite) disjoint union of all open convex cones $\Gamma(\mathcal{A})$ for all $\mathcal{A}$ in $\overline{\mathbb{A}}$.

Proposition 1.11. Let $P$ be $(M, \varepsilon)$-nondegenerate. Then the following are equivalent:

(i) $\beta$ in $\mathcal{N}^{n}$ is a positive combination of $\mathcal{A}$-that is, $\beta \in \Gamma(\mathcal{A})$;

(ii) the linear functional $f_{\beta}$ defined by $f_{\beta}(w)=\beta \cdot w$, when restricted to $\overline{\log (P)}$, reaches its maximum at all points and only at points of $\overline{\mathcal{F}(\mathcal{A})}$.

Proof. For (i) $\Rightarrow$ (ii), let $\mathcal{A}=\left\{\alpha_{1}, \ldots, \alpha_{k}\right\} \in \overline{\mathbb{A}}$ and assume there exist $\lambda_{1}, \ldots, \lambda_{k}>0$ such that $\beta=\sum_{j=1}^{k} \lambda_{j} \alpha_{j}$. Let $w$ be any point in $\overline{\log (P)}-\overline{\mathcal{F}(\mathcal{A})}$. From (1.8), we see that there must exist a $j \in\{1, \ldots, k\}$ such that $\alpha_{j} \cdot w<C_{\alpha_{j}}$; therefore,

$$
f_{\beta}(w)=\beta \cdot w=\sum_{j=1}^{k} \lambda_{j} \alpha_{j} \cdot w<\sum_{j=1}^{k} \lambda_{j} C_{\alpha_{j}} .
$$


But for $w \in \overline{\mathcal{F}(\mathcal{A})}$,

$$
f_{\beta}(w)=\sum_{j=1}^{k} \lambda_{j} C_{\alpha_{j}},
$$

so the result follows.

For (ii) $\Rightarrow$ (i), first note that $\mathcal{A}=\left\{\alpha_{1}, \ldots, \alpha_{k}\right\}=\left\{\alpha \in \mathcal{A}: \alpha \cdot w=C_{\alpha}\right.$ for all $w \in \overline{\mathcal{F}}\}$, and that $\mathcal{F}$ is non-empty. By Definition 1.10(ii), $\mathcal{A}$ is linearly independent. Thus, by solving the system

$$
\begin{gathered}
\alpha_{1} \cdot w=C_{\alpha_{1}} \\
\vdots \\
\alpha_{k} \cdot w=C_{\alpha_{k}},
\end{gathered}
$$

we have an $(n-k)$-dimensional linear affine space $\mathcal{W}$ containing $\mathcal{F}$. Let $w_{0}$ be a point in $\mathcal{F}$ satisfying Definition 1.10 (iii), and let $\beta_{k+1}, \ldots, \beta_{n}$ be a basis of $\mathcal{W}-w_{0}$ that is an $n-k$ dimensional vector space. Then we can express $\mathcal{W}$ as

$$
\mathcal{W}=\left\{w_{0}+\sum_{j=k+1}^{n} s_{j} \beta_{j}: s_{j} \in \mathbb{R}\right\},
$$

where

(a) $w_{0}+\sum_{j=k+1}^{n} s_{j} \beta_{j} \in \mathcal{F}=\mathcal{F}(\mathcal{A})$ for $\left|s_{j}\right|$ small;

(b) $\left\{\alpha_{1}, \ldots, \alpha_{k}, \beta_{k+1}, \ldots, \beta_{n}\right\}$ is a linear basis.

Thus, by (b), $\beta=\sum_{j=1}^{k} \lambda_{j} \alpha_{j}+\sum_{j=k+1}^{n} \lambda_{j} \beta_{j}$. But $f_{\beta}$ reaches its maximum at all points of $\overline{\mathcal{F}}$, and (a) implies that $\lambda_{j}=0$ for $j=k+1, \ldots, n$. For if not (say, $\lambda_{j}>0$ for some $j>k$ ) then we can find $w_{1}=w_{0}+s_{j} \beta_{j}$ where $s_{j}>0$ is so small that $w_{1} \in \mathcal{F}$. But then $f\left(w_{1}\right)>f\left(w_{0}\right)$, a contradiction. This means that $\beta$ is a linear combination of $\mathcal{A}$.

Suppose $\beta$ is not a nonnegative combination of $\mathcal{A}$. We can assume $\beta=$ $\sum_{j=1}^{k} \lambda_{j} \alpha_{j}, \lambda_{1}, \ldots, \lambda_{l} \geq 0$ and $\lambda_{l+1}, \ldots, \lambda_{k}<0$. But we can always find $w$ such that

$$
\begin{aligned}
\alpha_{1} \cdot w & =C_{\alpha_{1}} \\
& \vdots \\
\alpha_{l} \cdot w & =C_{\alpha_{l}} \\
\alpha_{l+1} \cdot w & <C_{\alpha_{l+1}} \\
& \vdots \\
\alpha_{k} \cdot w & <C_{\alpha_{k}} .
\end{aligned}
$$

It is easy to see that $w \in \overline{\log (P)}$ and $f_{\beta}(w) \gtrless f_{\beta}\left(w_{0}\right)$ for $w_{0} \in \mathcal{F}$, a contradiction.

Finally, suppose $\beta$ is not a positive combination of $\mathcal{A}$; then it is a positive combination of some $\mathcal{A}^{\prime} \subsetneq \mathcal{A}$. By the proof of (i) $\Rightarrow$ (ii), $f_{\beta}$ reaches its maximum on $\overline{\mathcal{F}\left(\mathcal{A}^{\prime}\right)} \supsetneq \overline{\mathcal{F}(\mathcal{A})}$, a contradiction.

Proposition 1.11 will allow us to represent all $\beta$ in $\mathcal{N}^{n}$ as a positive combination of a unique $\mathcal{A}$. We thus have the following result. 
Corollary 1.12. $\mathcal{N}^{n}$ can be decomposed into a finite disjoint union of open convex cones $\Gamma(\mathcal{A})$ for $\mathcal{A}$ in $\overline{\mathbb{A}}$. That is,

$$
\mathcal{N}^{n}=\bigcup_{\mathcal{A} \in \overline{\mathbb{A}}} \Gamma(\mathcal{A}) .
$$

Proof. For every $\beta$ in $\mathcal{N}^{n}, f_{\beta}$ must reach its maximum at all points of $\overline{\mathcal{F}}$ for some $\mathcal{F}$ in $\overline{\mathbb{F}}$. By Proposition $1.11, \beta$ is in $\Gamma(\mathcal{A})$ for some $\mathcal{A}$ in $\overline{\mathbb{A}}$. But $f_{\beta}$, as a linear functional, can reach its maximum on only one $\overline{\mathcal{F}}$, so $\mathcal{A}$ is unique.

Definition 1.13. We say $\mathcal{A} \in \overline{\mathbb{A}}$ is bounded (unbounded) if $\mathcal{F}(\mathcal{A})$ is bounded (unbounded).

Lemma 1.14. Let $\mathcal{A}=\left\{\alpha_{1}, \ldots, \alpha_{k}\right\}$ and $\alpha_{j}=\left(\alpha_{j}^{1}, \ldots, \alpha_{j}^{n}\right)$. Then $\mathcal{A}$ is unbounded if and only if there exists an $l \in\{1, \ldots, n\}$ such that $\alpha_{j}^{l}=0$ for all $j=$ $1, \ldots, k$.

Proof. $(\Rightarrow)$ Since $P$ is bounded, for a face $\mathcal{F}$ in $\overline{\log (P)}$ to be unbounded there must exist an $l \in\{1, \ldots, n\}$ such that if $w_{0}=\left(w_{0}^{1}, \ldots, w_{0}^{n}\right) \in \mathcal{F}$ then

$$
\left(w_{0}^{1}, \ldots, w^{j}, \ldots, w_{0}^{n}\right) \in \mathcal{F} \text { for all } w^{j}<w_{0}^{j} .
$$

For Remark 1.1 shows that if $P$ is bounded and $w=\left(w^{1}, \ldots, w^{n}\right) \in \overline{\log (P)}$, then every component $w^{j}$ is bounded above. Thus, from Definition 1.7, we have $\alpha_{j}^{l}=$ 0 for all $j=1, \ldots, k$.

$(\Leftarrow)$ From Definition 1.7 , if there exists an $l \in\{1, \ldots, n\}$ such that $\alpha_{j}^{l}=0$ for all $j=1, \ldots, k$, then $\mathcal{F}$ must be unbounded.

Let us define

$$
\mathbb{F}=\{\mathcal{F} \subseteq \overline{\log (P)}: \mathcal{F} \text { is a non-empty bounded face }\}
$$

and

$$
\mathbb{A}=\{\mathcal{A} \subseteq \mathcal{B}: \mathcal{F}(\mathcal{A}) \text { is a non-empty bounded face }\} .
$$

Then we have the following.

Proposition 1.15.

$$
\mathbb{N}^{n}=\bigcup_{\mathcal{A} \in \mathbb{A}} \Gamma(\mathcal{A}) .
$$

Moreover, no components of elements in $\mathcal{A} \in \mathbb{A}$ will be simultaneously zero.

Proof. If $\mathcal{A} \in \overline{\mathbb{A}}-\mathbb{A}$ then by Lemma 1.14 , for all $\beta=\left(b^{1}, \ldots, b^{n}\right) \in \Gamma(\mathcal{A})$ there exists an $l \in\{1, \ldots, n\}$ such that $b^{l}=0$, 一 that is $\beta \in \mathcal{N}^{n}-\mathbb{N}^{n}$. Therefore, $\Gamma(\mathcal{A}) \subseteq \mathcal{N}^{n}-\mathbb{N}^{n}$ and

$$
\bigcup_{\mathcal{A} \in \overline{\mathbb{A}}-\mathbb{A}} \Gamma(\mathcal{A}) \subseteq \mathcal{N}^{n}-\mathbb{N}^{n} .
$$

But for $\beta=\left(b^{1}, \ldots, b^{n}\right) \in \mathcal{N}^{n}-\mathbb{N}^{n}$ there exists an $l \in\{1, \ldots, n\}$ such that $b^{l}=0$, and by Corollary 1.12 there exists an $\mathcal{A}$ such that $\beta \in \Gamma(\mathcal{A})$. Yet this 
means that $\beta$ is a positive combination of $\mathcal{A}$, so if $\mathcal{A}=\left\{\alpha_{1}, \ldots, \alpha_{k}\right\}$ and $\alpha_{j}=$ $\left(\alpha_{j}^{1}, \ldots, \alpha_{j}^{n}\right)$ then $\alpha_{j}^{l}=0$ for all $j=1, \ldots, k$. By Lemma $1.14, \mathcal{A} \in \overline{\mathbb{A}}-\mathbb{A}$. Therefore,

$$
\bigcup_{\mathcal{A} \in \mathbb{A}-\mathbb{A}} \Gamma(\mathcal{A}) \supseteq \mathcal{N}^{n}-\mathbb{N}^{n}
$$

This shows

$$
\bigcup_{\mathcal{A} \in \mathbb{\mathbb { A }}-\mathbb{A}} \Gamma(\mathcal{A})=\mathcal{N}^{n}-\mathbb{N}^{n}
$$

Now using Corollary 1.12 again, the result follows.

\section{Estimates for $L^{2}$-norms of $z^{\beta}$}

We wish to calculate the Bergman kernel for $P$. Since a monomial polyhedron is a Reinhardt domain, by letting $\overline{\mathbf{1}}=(1, \ldots, 1)$ and $\zeta_{\beta}(z)=z^{\beta}$ we have

$$
K_{P}\left(z_{0}, w_{0}\right)=\sum_{\beta \in \mathcal{N}^{n}} \frac{z_{0}^{\beta} \bar{w}_{0}^{\beta}}{\left\|\zeta_{\beta}\right\|^{2}}=\sum_{\beta+\overline{\mathbf{i}} \in \mathbb{N}^{n}} \frac{z_{0}^{\beta} \bar{w}_{0}^{\beta}}{\left\|\zeta_{\beta}\right\|^{2}}=\sum_{\mathcal{A} \in \mathbb{A}} \sum_{\beta+\overline{\mathbf{i}} \in \Gamma(\mathcal{A})} \frac{z_{0}^{\beta} \bar{w}_{0}^{\beta}}{\left\|\zeta_{\beta}\right\|^{2}} .
$$

Note that the first summation in the last expression is a finite sum.

Let $\beta+\overline{\mathbf{1}}$ be in $\Gamma(\mathcal{A})$ for some $\mathcal{A}$ in $\mathbb{A}$, and let $w_{\beta+\overline{\mathbf{1}}}$ be a point on $\mathcal{F}=\mathcal{F}(\mathcal{A})$. Define

$$
S_{\beta+\overline{\mathbf{1}}}(t)=\overline{\log (P)} \cap\left\{w:(\beta+\overline{\mathbf{1}}) \cdot w=(\beta+\overline{\mathbf{1}}) \cdot w_{\beta+\overline{\mathbf{1}}}-t\right\} .
$$

Since the function $e^{2(\beta+\overline{\mathbf{1}}) \cdot w}$ is a constant on $S_{\beta+\overline{\mathbf{1}}}(t)$ for fixed $t$, if we define $A_{\beta+\overline{\mathbf{1}}}(t)$ to be the function measuring the $(n-1)$-dimensional area of $S_{\beta+\overline{\mathbf{1}}}(t)$ then

$$
\begin{aligned}
\left\|\zeta_{\beta}\right\|^{2} & =\int_{P}\left|z^{\beta}\right|^{2} d V(z) \\
& =(2 \pi)^{n} \int_{\log (P)} e^{2(\beta+\overline{\mathbf{1}}) \cdot w} d V(w) \\
& =\frac{(2 \pi)^{n}}{\|\beta+\overline{\mathbf{1}}\|} \int_{0}^{\infty} e^{2(\beta+\overline{\mathbf{1}}) \cdot w_{\beta+\overline{\mathbf{1}}}-2 t} A_{\beta+\overline{\mathbf{1}}}(t) d t .
\end{aligned}
$$

The last equality is gained by performing a unitary change of coordinates so that $d t=d(\beta+\overline{\mathbf{1}})$, where $\beta+\overline{\mathbf{1}}=\left(\beta_{1}+1, \ldots, \beta_{n}+1\right)$.

For the convenience of discussion, let us use the following notation:

$$
\begin{aligned}
\Omega_{\beta+\overline{\mathbf{1}}}(t) & =\overline{\log (P)} \cap\left\{w:(\beta+\overline{\mathbf{1}}) \cdot w \geq(\beta+\overline{\mathbf{1}}) \cdot w_{\beta+\overline{\mathbf{1}}}-t\right\} ; \\
\Delta_{\beta+\overline{\mathbf{1}}}(\delta) & =\left\{w: w=w_{\beta+\overline{\mathbf{1}}}+s \cdot\left(w^{\prime}-w_{\beta+\overline{\mathbf{1}}}\right) \text { for all } w^{\prime} \in S_{\beta+\overline{\mathbf{1}}}(\delta) \text { and } s \geq 0\right\} ; \\
\Delta_{\beta+\overline{\mathbf{1}}}(\delta, t) & =\Delta_{\beta+\overline{\mathbf{1}}}(\delta) \cap\left\{w:(\beta+\overline{\mathbf{1}}) \cdot w \geq(\beta+\overline{\mathbf{1}}) \cdot w_{\beta+\overline{\mathbf{1}}}-t\right\} .
\end{aligned}
$$

Note that $\Delta_{\beta+\overline{\mathbf{1}}}(\delta)=\Delta_{\beta+\overline{\mathbf{1}}}(\delta, \infty)$. 
Now notice

$$
\begin{gathered}
\int_{\log (P)}=\int_{\Omega_{\beta+\overline{\mathbf{1}}}(\delta)}+\int_{\log (P)-\Omega_{\beta+\overline{\mathbf{1}}}(\delta)}, \\
\int_{\Omega_{\beta+\overline{\mathbf{1}}}(\delta)} \geq \int_{\Delta_{\beta+\overline{\mathbf{1}}}(\delta, \delta)}, \quad \text { and } \int_{\Delta_{\beta+\overline{\mathbf{1}}}(\delta)-\Delta_{\beta+\overline{\mathbf{1}}}(\delta, \delta)} \geq \int_{\log (P)-\Omega_{\beta+\overline{\mathbf{1}}}(\delta)} .
\end{gathered}
$$

The inequalities are obtained from

$$
\Omega_{\beta+\overline{\mathbf{1}}}(\delta) \supseteq \Delta_{\beta+\overline{\mathbf{1}}}(\delta, \delta) \quad \text { and } \quad \Delta_{\beta+\overline{\mathbf{1}}}(\delta)-\Delta_{\beta+\overline{\mathbf{1}}}(\delta, \delta) \supseteq \log (P)-\Omega_{\beta+\overline{\mathbf{1}}}(\delta),
$$

which in turn are results of the convexity of $\log (P)$. The first inclusion is easy to show. For the second inclusion, suppose $w \in \log (P)-\Omega_{\beta+\overline{\mathbf{1}}}(\delta)$; the line segment between $w$ and $w_{\beta+\overline{\mathbf{1}}}$ must be in $\log (P)$, which intersects $S_{\beta+\overline{\mathbf{1}}}(\delta)$ at one point. Thus $w$ is a point in $\Delta_{\beta+\overline{\mathbf{1}}}(\delta)$, but it cannot be in $\Delta_{\beta+\overline{\mathbf{1}}}(\delta, \delta)$. The result follows.

If we can show there exists a constant $c=c(\delta)>0$ such that

$$
\int_{\Delta_{\beta+\overline{\mathbf{1}}}(\delta, \delta)} \geq c \int_{\Delta_{\beta+\overline{\mathbf{1}}}(\delta)-\Delta_{\beta+\overline{\mathbf{1}}}(\delta, \delta)},
$$

then by

$$
\left(1+\frac{1}{c}\right) \int_{\Omega_{\beta+\overline{\mathbf{1}}}(\delta)} \geq \int_{\Omega_{\beta+\overline{\mathbf{1}}}(\delta)}+\int_{\log (P)-\Omega_{\beta+\overline{\mathbf{1}}}(\delta)}=\int_{\log (P)} \geq \int_{\Omega_{\beta+\overline{\mathbf{1}}}(\delta)}
$$

we have

$$
\int_{\log (P)} \approx \int_{\Omega_{\beta+\overline{\mathbf{1}}}(\delta)}
$$

To prove (2.2), we will instead show that there exists a constant $c>0$ such that

$$
\int_{\Delta_{\beta+\mathbf{1}}(\delta, \delta)} \geq c \int_{\Delta_{\beta+\overline{\mathbf{1}}}(\delta)} .
$$

In general, however,

$$
\begin{aligned}
\int_{\Delta_{\beta+\overline{\mathbf{1}}}(\delta, s)} e^{2(\beta+\overline{\mathbf{1}}) \cdot w} d V(w) & =\int_{0}^{s} e^{2(\beta+\overline{\mathbf{1}}) \cdot w_{\beta+\overline{\mathbf{1}}}-2 t} A_{\beta+\overline{\mathbf{1}}}(\delta)\left(\frac{t}{\delta}\right)^{n-1} d t \\
& =\frac{e^{2(\beta+\overline{\mathbf{1}}) \cdot w_{\beta+\overline{\mathbf{1}}} A_{\beta+\overline{\mathbf{1}}}(\delta)}}{\delta^{n-1}} \int_{0}^{s} e^{-2 t} t^{n-1} d t
\end{aligned}
$$

Set

$$
f(s)=\int_{0}^{s} e^{-2 t} t^{n-1} d t
$$

and observe that

$$
f(\delta)>\frac{(n-1) !}{2^{n}} \cdot \frac{(2 \delta)^{n}}{e^{2 \delta} \cdot n !} \text { and } \lim _{s \rightarrow \infty} f(s)=\frac{(n-1) !}{2^{n}} .
$$

With $c(\delta)=(2 \delta)^{n} /\left(e^{2 \delta} \cdot n !\right)$, we have 


$$
\int_{\Delta_{\beta+\overline{\mathbf{1}}}(\delta, \delta)} e^{2(\beta+\overline{\mathbf{1}}) \cdot w} d V(w)>c(\delta) \int_{\Delta_{\beta+\overline{\mathbf{1}}}(\delta)} e^{2(\beta+\overline{\mathbf{1}}) \cdot w} d V(w) .
$$

Therefore, by (2.3), we can state the following proposition.

Proposition 2.1. With $c=c(\delta)=(2 \delta)^{n} /\left(e^{2 \delta} \cdot n !\right)$, we have

$$
\int_{\Omega_{\beta+\overline{\mathbf{1}}}(\delta)} e^{2(\beta+\overline{\mathbf{1}}) \cdot w} d V(w) \leq\left\|z^{\beta}\right\|^{2} \leq\left(1+\frac{1}{c}\right) \int_{\Omega_{\beta+\overline{\mathbf{1}}}(\delta)} e^{2(\beta+\overline{\mathbf{1}}) \cdot w} d V(w) .
$$

Moreover,

$$
\int_{\Omega_{\beta+\overline{\mathbf{1}}}(\delta)} e^{2(\beta+\overline{\mathbf{1}}) \cdot w} d V(w)=\frac{e^{2(\beta+\overline{\mathbf{1}}) \cdot w_{\beta+\overline{\mathbf{1}}}}}{\|\beta+\overline{\mathbf{1}}\|} \int_{0}^{\delta} e^{-2 t} A_{\beta+\overline{\mathbf{1}}}(t) d t .
$$

Notice that Proposition 2.1 is true for all Reinhardt domains, not necessarily monomial polyhedrons.

In order to sum (2.1) we must take a closer look at $A_{\beta+\overline{\mathbf{1}}}(t)$ for $t \in[0, \delta]$, where $\delta=\delta(M, \varepsilon)$ will be determined later, and apply certain elementary linear algebraic computation to carry out a formula for $A_{\beta+\overline{\mathbf{1}}}(t)$ and thus $\left\|z^{\beta}\right\|^{2}$. Here we will make use of the properties of monomial polyhedra and Definition 1.10 of $(M, \varepsilon)$-nondegeneracy.

Let $\beta+\overline{\mathbf{1}} \in \Gamma(\mathcal{A})$ for some $\mathcal{A}=\left\{\alpha_{1}, \ldots, \alpha_{k}\right\}$ in $\mathbb{A}$, let $\mathcal{F}=\mathcal{F}(\mathcal{A})$, and assume that $A^{n-k}(\mathcal{F})$ is the area of $\mathcal{F}$ measured as an $(n-k)$-dimensional object. Notice that $A^{n-k}(\mathcal{F})$ is never zero (by the definition of $(M, \varepsilon)$-nondegeneracy) whereas $A_{\beta+\overline{\mathbf{1}}}(0)$, measuring the same face $\mathcal{F}$ as a $(n-1)$-dimensional object, is usually zero unless $|\mathcal{A}(\mathcal{F})|=1$.

Our purpose for the rest of this section is to show that

$$
A_{\beta+\overline{\mathbf{1}}}(t) \approx \frac{\|\beta+\overline{\mathbf{1}}\| t^{k-1} \cdot A^{n-k}(\mathcal{F})}{\lambda_{1} \cdots \lambda_{k}} .
$$

Combining (2.4) and (2.5), this implies

$$
\left\|\zeta_{\beta}\right\|^{2}=\int_{P}\left|z^{\beta}\right|^{2} d V(z) \approx \frac{e^{2(\beta+\overline{\mathbf{1}}) \cdot w_{\beta+\overline{\mathbf{1}}} \cdot A^{n-k}(\mathcal{F})}}{\lambda_{1} \cdots \lambda_{k}},
$$

where the ratio depends only on $M, \varepsilon$, and $n$.

First let us simplify the domain. Let

$$
\mathbb{A}(\mathcal{A})=\left\{\mathcal{A}^{\prime} \in \mathbb{A}: \mathcal{A} \subset \mathcal{A}^{\prime}\right\}
$$

and

$$
\mathcal{B}(\mathcal{A})=\bigcup_{\mathcal{A}^{\prime} \in \mathbb{A}(\mathcal{A})} \mathcal{A}^{\prime}=\left\{\alpha_{1}, \ldots, \alpha_{k}, \alpha_{k+1}, \ldots, \alpha_{k+m}\right\} .
$$

Notice that the boundary of $\mathcal{F}(\mathcal{A})$ is a union of all faces $\mathcal{F}\left(\mathcal{A}^{\prime}\right)$, where $\mathcal{A} \subsetneq \mathcal{A}^{\prime} \in$ $\mathbb{A}(\mathcal{A})$.

By a unitary change of coordinates, we can assume $\alpha_{j}=\left(\alpha_{j, 1}, \ldots, \alpha_{j, j}\right.$, $0, \ldots, 0)$ for $j=1, \ldots, k$. Thus $\mathcal{F}$ will be defined by 


$$
\begin{aligned}
& \alpha_{1} \cdot w=0 \\
& \vdots \\
& \alpha_{k} \cdot w=0 \\
& \alpha_{k+1} \cdot w<C_{1} \\
& \vdots \\
& \alpha_{k+m} \cdot w<C_{m},
\end{aligned}
$$

and $\log (P)$ around $\mathcal{F}$ will be defined by

$$
\begin{aligned}
\alpha_{1} \cdot w & <0 \\
& \vdots \\
\alpha_{k} \cdot w & <0 \\
\alpha_{k+1} \cdot w & <C_{1} \\
& \vdots \\
\alpha_{k+m} \cdot w & <C_{m} .
\end{aligned}
$$

By writing all elements $x$ in $\mathbb{R}^{n}$ into $x^{\prime}$ in $\mathbb{R}^{k}$ and $x^{\prime \prime}$ in $\mathbb{R}^{n-k}$, where $x^{\prime}$ consists of the first $k$ components while $x^{\prime \prime}$ consists of the rest, we can see that

$$
\begin{aligned}
\alpha_{1} \cdot w & =\alpha_{1}^{\prime} \cdot w^{\prime} \\
& \vdots \\
\alpha_{k} \cdot w & =\alpha_{k}^{\prime} \cdot w^{\prime}
\end{aligned}
$$

and that $\Omega_{\beta+\overline{\mathbf{1}}}(t)$, for $t \in[0, \delta]$, is defined by

$$
\begin{aligned}
(\beta+\overline{\mathbf{1}})^{\prime} \cdot w^{\prime} & >-t \\
\alpha_{1}^{\prime} \cdot w^{\prime} & <0 \\
& \vdots \\
\alpha_{k}^{\prime} \cdot w^{\prime} & <0
\end{aligned}
$$

and

$$
\begin{aligned}
\alpha_{k+1}^{\prime \prime} \cdot w^{\prime \prime} & <C_{1}-\alpha_{k+1}^{\prime} \cdot w^{\prime} \\
& \vdots \\
\alpha_{k+m}^{\prime \prime} \cdot w^{\prime \prime} & <C_{m}-\alpha_{k+m}^{\prime} \cdot w^{\prime} .
\end{aligned}
$$

Note that $(\beta+\overline{\mathbf{1}}) \cdot w=(\beta+\overline{\mathbf{1}})^{\prime} \cdot w^{\prime}$.

For each $w^{\prime}$ satisfying (2.6), define $\mathcal{W}\left(w^{\prime}\right)=\left\{w^{\prime \prime}:\left(w^{\prime}, w^{\prime \prime}\right) \in \log (P)\right\}$. We know that when $w^{\prime}=0$, the set of all possible $w^{\prime \prime}$ satisfying system (2.7) (i.e., $\mathcal{W}(0))$ is exactly $\mathcal{F}$, and we want to understand by how much the volume of $\mathcal{W}\left(w^{\prime}\right)$ can vary when $w^{\prime}$ changes.

The possible values that $w^{\prime}$ can take are controlled only by the system of inequalities (2.6) for $t \in[0, \delta]$, which defines a $k$-simplex. Since all inequalities involved in (2.7) are linear, we know the maximal change of the volume of $\mathcal{W}\left(w^{\prime}\right)$ happens on the extreme points of the $k$-simplex defined by (2.6) with $t \in[0, \delta]$. 
The extreme points are either 0 or the solution to the system of equations

$$
\begin{aligned}
\alpha_{1}^{\prime} \cdot\left(w^{j}\right)^{\prime} & =0 \\
\vdots & \\
\alpha_{j-1}^{\prime} \cdot\left(w^{j}\right)^{\prime} & =0 \\
(\beta+\overline{\mathbf{1}})^{\prime} \cdot\left(w^{j}\right)^{\prime} & =-t \\
\alpha_{j+1}^{\prime} \cdot\left(w^{j}\right)^{\prime} & =0 \\
\vdots & \\
\alpha_{k}^{\prime} \cdot\left(w^{j}\right)^{\prime} & =0
\end{aligned}
$$

for $j=1, \ldots, k$. By plugging in $\beta+\overline{\mathbf{1}}=\sum_{j=1}^{k} \lambda_{j} \alpha_{j}$ and writing $[\alpha]_{k}=\left[\alpha_{i, j}\right]$, $i, j=1, \ldots, k$, we have a unique solution

$$
\left(w^{j}\right)^{\prime}=[\alpha]_{k}^{-1}\left(\frac{-t}{\lambda_{j}}\right) e_{j}, \quad \text { where } e_{j}=\left[\begin{array}{c}
0 \\
\vdots \\
1 \\
\vdots \\
0
\end{array}\right] .
$$

But plugging this information back into

$$
\begin{aligned}
\alpha_{k+1}^{\prime \prime} \cdot w^{\prime \prime} & <C_{1}-\alpha_{k+1}^{\prime} \cdot w^{\prime} \\
& \vdots \\
\alpha_{k+m}^{\prime \prime} \cdot w^{\prime \prime} & <C_{m}-\alpha_{k+m}^{\prime} \cdot w^{\prime}
\end{aligned}
$$

(system (2.7)) when $\alpha_{k+i}^{\prime}=\sum_{j=1}^{k} \lambda_{i, j} \alpha_{j}$, we have

$$
\alpha_{k+i}^{\prime} \cdot\left(w^{j}\right)^{\prime}=\frac{-t \lambda_{i, j}}{\lambda_{j}} .
$$

Thus, for all $j=1, \ldots, k$,

$$
\begin{aligned}
\alpha_{k+1}^{\prime \prime} \cdot w^{\prime \prime} & <C_{1}+t \lambda_{1, j} / \lambda_{j} \\
& \vdots \\
\alpha_{k+m}^{\prime \prime} \cdot w^{\prime \prime} & <C_{m}+t \lambda_{m, j} / \lambda_{j} .
\end{aligned}
$$

Notice that $\alpha$ has positive integer components in the original coordinates and, by Definition 1.10(i), the sum of components of $\alpha$ is less than $M$. Hence $\lambda_{j}$ is positive and bounded away from 0 by a constant depending only on $M$, and $\lambda_{i, j}$ is positive and bounded by $M$. Thus there exist $C=C(M)>0$ such that

$$
\left|\frac{\lambda_{i, j}}{\lambda_{j}}\right|<C(M), \quad i=1, \ldots, m, j=1, \ldots, k .
$$

Next, using Definition 1.10(iii) on $\mathcal{F}$ (where $\mathcal{F}$ can be expressed by the system (2.7) when $w^{\prime}=0$ ) and taking $w^{\prime \prime}=\varepsilon \cdot \alpha_{k+i}^{\prime \prime} /\left\|\alpha_{k+i}^{\prime \prime}\right\|$, since $\left\|w^{\prime \prime}\right\|=\varepsilon$ and $w^{\prime \prime} \in$ $\overline{\mathcal{F}}$ we have $\varepsilon \cdot\left\|\alpha_{k+i}^{\prime \prime}\right\| \leq C_{i}$. But $\left\|\alpha_{k+i}^{\prime \prime}\right\| \geq c$ for some $c=c(M)>0$. Therefore, $C_{i} \geq \varepsilon \cdot c(M)$. 
Now set $\delta=\varepsilon c(M) / 2 C(M)$, define $\mathcal{F}_{d}=\left\{w^{\prime \prime} \in \mathbb{R}^{n-k}: \alpha_{k+i}^{\prime \prime} \cdot w^{\prime \prime}<d \cdot C_{i}\right.$, $i=1, \ldots, m\}$, and define $L_{\beta+\overline{\mathbf{1}}}(t)=\left\{w^{\prime} \in \mathbb{R}^{n}: \alpha_{j}^{\prime} \cdot w^{\prime}<0\right.$ for $j=1, \ldots, k$ and $\left.(\beta+\overline{\mathbf{1}})^{\prime} \cdot w^{\prime}=-t\right\}$. First, notice $\mathcal{F}=\mathcal{F}_{1}$. We also have

$$
L_{\beta+\overline{\mathbf{1}}}(t) \times \mathcal{F}_{1 / 2} \subseteq S_{\beta+\overline{\mathbf{1}}}(t) \subseteq L_{\beta+\overline{\mathbf{1}}}(t) \times \mathcal{F}_{2} \text { for } t \in[0, \delta] .
$$

Now let $P_{\beta+\overline{\mathbf{1}}}(\delta)=\bigcup_{t \in[0, \delta]} L_{\beta+\overline{\mathbf{1}}}(t)$. Then $P_{\beta+\overline{\mathbf{1}}}(\delta)$ is a $k$-simplex with extreme points at 0 and $\left(w^{j}\right)^{\prime}$ for $j=1, \ldots, k$, where $\left(w^{j}\right)^{\prime}=[\alpha]_{k}^{-1}\left(-t / \lambda_{j}\right) e_{j}$. But for any $k$-simplex with extreme points at 0 and $a_{i}=\left(a_{i, j}\right), i, j=1, \ldots, k$, the volume is exactly $\frac{1}{k !} \operatorname{det}\left[a_{i, j}\right]$. Thus, the volume of $P_{\beta+\overline{\mathbf{1}}}(t)$ is

$$
A^{k}\left(P_{\beta+\overline{\mathbf{1}}}(t)\right)=\frac{t^{k}}{k ! \cdot \operatorname{det}[\alpha]_{k} \cdot \lambda_{1} \cdots \lambda_{k}} .
$$

However,

$$
A^{k}\left(P_{\beta+\overline{\mathbf{1}}}(t)\right)=\frac{1}{\|\beta+\overline{\mathbf{1}}\|} \int_{0}^{t} A^{k-1}\left(L_{\beta+\overline{\mathbf{1}}}(\eta)\right) d \eta .
$$

By taking derivatives with respect to $t$ on both ends of the preceding equations, we have the volume of $L_{\beta+\overline{\mathbf{1}}}(t)$ as

$$
A^{k-1}\left(L_{\beta+\overline{\mathbf{1}}}(t)\right)=\frac{\|\beta+\overline{\mathbf{1}}\| \cdot t^{k-1}}{(k-1) ! \cdot \operatorname{det}[\alpha]_{k} \cdot \lambda_{1} \cdots \lambda_{k}} .
$$

Also note that

$$
A^{n-k}\left(\mathcal{F}_{d}\right)=d^{n-k} \cdot A^{n-k}\left(\mathcal{F}_{1}\right), \quad \text { where } \mathcal{F}_{1}=\mathcal{F} .
$$

Thus, by (2.8), for $t \in[0, \delta]$ we have

$$
\begin{aligned}
\frac{2^{-n} \cdot\|\beta+\overline{\mathbf{1}}\| \cdot t^{k-1} \cdot A^{n-k}(\mathcal{F})}{(k-1) ! \cdot \operatorname{det}[\alpha]_{k} \cdot \lambda_{1} \cdots \lambda_{k}} & \leq A_{\beta+\overline{\mathbf{1}}}(t) \\
& \leq \frac{2^{n} \cdot\|\beta+\overline{\mathbf{1}}\| \cdot t^{k-1} \cdot A^{n-k}(\mathcal{F})}{(k-1) ! \cdot \operatorname{det}[\alpha]_{k} \cdot \lambda_{1} \cdots \lambda_{k}}
\end{aligned}
$$

or simply

$$
A_{\beta+\overline{\mathbf{1}}}(t) \approx \frac{\|\beta+\overline{\mathbf{1}}\| \cdot t^{k-1} \cdot A^{n-k}(\mathcal{F})}{(k-1) ! \cdot \operatorname{det}[\alpha]_{k} \cdot \lambda_{1} \cdots \lambda_{k}},
$$

where the ratio depends only on the total dimension $n$.

Combining (2.4), (2.5), and (2.9) with $\zeta_{\beta}(z)=z^{\beta}$, we have

$$
\begin{aligned}
\left\|\zeta_{\beta}\right\|^{2} & \approx \frac{e^{2\left(\beta+\overline{\mathbf{1}} \cdot w_{\beta+\mathbf{1}}\right.} \cdot A^{n-k}(\mathcal{F})}{(k-1) ! \cdot \operatorname{det}[\alpha]_{k} \cdot \lambda_{1} \cdots \lambda_{k}} \int_{0}^{\delta} e^{-2 t} t^{k-1} d t \\
& \approx \frac{e^{2(\beta+\overline{\mathbf{1}}) \cdot w_{\beta+\overline{\mathbf{1}}}} \cdot A^{n-k}(\mathcal{F})}{\operatorname{det}[\alpha]_{k} \cdot \lambda_{1} \cdots \lambda_{k}}
\end{aligned}
$$

where the first approximation depends only on $n$ while the second approximation depends on $n$ and $\delta$, which in turn is defined as a function of $M$ and $\varepsilon$. 
Let us summarize in the form of a proposition.

Proposition 2.2. Let $\log (P)$ be $(M, \varepsilon)$-nondegenerate. For any $\beta$ in $\mathcal{N}^{n}$, there exists a unique $\mathcal{A}$ in $\mathbb{A}$ such that $\beta+\overline{\mathbf{1}}$ is in $\Gamma(\mathcal{A})$. For any $w_{\beta+\overline{\mathbf{1}}}$ in $\mathcal{F}=\mathcal{F}(\mathcal{A})$ we have, with the ratio depending on $M, \varepsilon$, and $n$,

$$
\left\|z^{\beta}\right\|^{2} \approx \frac{e^{2(\beta+\overline{\mathbf{1}}) \cdot w_{\beta+\overline{\mathbf{1}}}} \cdot A^{n-k}(\mathcal{F})}{\operatorname{det}[\alpha]_{k} \cdot \lambda_{1} \cdots \lambda_{k}},
$$

where $A^{n-k}(\mathcal{F})$ is the $(n-k)$-dimensional volume of $\mathcal{F}$. When $k=n, A^{0}(\mathcal{F})=1$.

REMARK 2.1. Because $(\beta+\overline{\mathbf{1}}) \cdot w$ is constant for $w$ in $\mathcal{F}$, it does not matter which $w_{\beta+\overline{1}}$ we choose in $\mathcal{F}$ for Proposition 2.2. Also, the log term we usually see in the Bergman kernel will come out naturally from the calculation of the term $A^{n-k}(\mathcal{F})$ when $k<n$.

\section{Estimate for the Bergman Kernel on a Diagonal}

Using (2.1) on the diagonal with $\zeta_{\beta}(z)=z^{\beta}$, we have

$$
K_{P}\left(z_{0}, z_{0}\right)=\sum_{\mathcal{A} \in \mathbb{A}} \sum_{\beta+\overline{\mathbf{1}} \in \Gamma(\mathcal{A})} \frac{\left|z_{0}^{\beta}\right|^{2}}{\left\|\zeta_{\beta}\right\|^{2}} .
$$

The first summation is a finite sum, for there are only finitely many $\mathcal{A}$ in $\mathbb{A}$. We want to express the second one as a finite sum, too, by considering the fundamental $\operatorname{set} \mathcal{U}(\mathcal{A})$ of the open cone $\Gamma(\mathcal{A})$, where

$$
\mathcal{U}(\mathcal{A})=\Gamma(\mathcal{A}) \cap\left\{\alpha: \alpha=\sum_{j=1}^{k} \lambda_{j} \alpha_{j}, 0<\lambda_{j} \leq 1\right\} .
$$

Notice that $\mathcal{U}(\mathcal{A})$ contains only finitely many indices, and that the open cone $\Gamma(\mathcal{A})$ can be decomposed into the fundamental set $\mathcal{U}(\mathcal{A})$ and its integral multiple translations $\mathcal{U}_{\left(m_{1}, \ldots, m_{n}\right)}(\mathcal{A})$, where

$$
\mathcal{U}_{\left(m_{1}, \ldots, m_{k}\right)}(\mathcal{A})=\sum_{j=1}^{k} m_{j} \alpha_{j}+\mathcal{U}(\mathcal{A})
$$

for $m_{j}=0,1,2, \ldots(j=1, \ldots, k)$. That is, for $\beta+\overline{\mathbf{1}} \in \Gamma(\mathcal{A})$, there exist nonnegative integers $m_{1}, \ldots, m_{k}$ and nonnegative numbers $\lambda_{1}, \ldots, \lambda_{k}$ such that $0<$ $\lambda_{j} \leq 1$ and

$$
\beta+\overline{\mathbf{1}}=\sum_{j=1}^{k}\left(m_{j}+\lambda_{j}\right) \alpha_{j} .
$$

But notice that, since all components in $\beta+\overline{\mathbf{1}}$ take positive integer values and the sum of components for all elements in $\mathcal{A}$ is bounded by $M$, the value for $\lambda_{j}$ $(j=1, \ldots, k)$ is bounded away from 0 where the lower bound depends only on $M$. Thus we have, with the ratio depending only on $M$,

$$
m_{j}+\lambda_{j} \approx m_{j}+1
$$


By letting $w_{0}=\log \left|z_{0}\right|$ and $z_{\mathcal{F}}=e^{w_{\mathcal{F}}}$, where $w_{\mathcal{F}}$ is any point on $\mathcal{F}=\mathcal{F}(\mathcal{A})$, we have

$$
\begin{aligned}
& \sum_{\beta+\overline{\mathbf{1}} \in \Gamma(\mathcal{A})} \frac{\left|z_{0}^{\beta}\right|^{2}}{\left\|z^{\beta}\right\|^{2}} \\
& \quad \approx \sum_{m_{1}=0}^{\infty} \cdots \sum_{m_{k}=0}^{\infty} \sum_{\beta+\overline{\mathbf{1}} \in \mathcal{U}(\mathcal{A})} \frac{\left(m_{1}+1\right) \cdots\left(m_{k}+1\right) \cdot\left|z_{0}^{\beta+\sum_{j=1}^{k} m_{j} \alpha_{j}}\right|^{2}}{\left|z_{\mathcal{F}}^{(\beta+\overline{\mathbf{1}})+\sum_{j=1}^{k} m_{j} \alpha_{j}}\right|^{2} \cdot A^{n-k}(\mathcal{F})} \\
& \approx \prod_{j=1}^{k}\left(\sum_{m=0}^{\infty}(m+1) e^{2 m \alpha_{j} \cdot\left(w_{0}-w_{\mathcal{F}}\right)}\right) \cdot \sum_{\beta+\overline{\mathbf{1}} \in \mathcal{U}(\mathcal{A})} \frac{\left|z_{0}^{\beta}\right|^{2}}{\left\|z^{\beta}\right\|^{2}} \\
& =\prod_{j=1}^{k} \frac{1}{\left(1-e^{2 \alpha_{j} \cdot\left(w_{0}-w_{\mathcal{F}}\right)}\right)^{2}} \cdot \sum_{\beta+\overline{\mathbf{1}} \in \mathcal{U}(\mathcal{A})} \frac{\left|z_{0}^{\beta}\right|^{2}}{\left\|z^{\beta}\right\|^{2}} \\
& =\prod_{j=1}^{k} \frac{1}{\left(1-\left|\left(z_{0} / z_{\mathcal{F}}\right)^{\alpha_{j}}\right|^{2}\right)^{2}} \cdot \sum_{\beta+\overline{\mathbf{1}} \in \mathcal{U}(\mathcal{A})} \frac{\left|z_{0}^{\beta}\right|^{2}}{\left\|z^{\beta}\right\|^{2}} \\
& \quad \approx \prod_{j=1}^{k} \frac{1}{\sum_{\beta+\overline{\mathbf{1}} \in \mathcal{U}(\mathcal{A})} \frac{\left|z_{0}^{\beta}\right|^{2}}{\left\|z^{\beta}\right\|^{2}} .}
\end{aligned}
$$

Finally by (3.1), we have the following result.

THEOREM 3.1. Let $P$ be an $(M, \varepsilon)$-nondegenerate bounded monomial polyhedron, and let $\zeta_{\beta}(z)=z^{\beta}$. Then for $\mathcal{A}=\left\{\alpha_{1}, \ldots, \alpha_{k}\right\}$ we have

$$
K_{P}\left(z_{0}, z_{0}\right) \approx \sum_{\mathcal{A} \in \mathbb{A}}\left(\prod_{j=1}^{k} \frac{1}{\left(1-\left|\left(z_{0} / z_{\mathcal{F}}\right)^{\alpha_{j}}\right|\right)^{2}} \cdot \sum_{\beta+\overline{\mathbf{1}} \in \mathcal{U}(\mathcal{A})} \frac{\left|z_{0}^{\beta}\right|^{2}}{\left\|\zeta_{\beta}\right\|^{2}}\right),
$$

with the ratio depending only on $M, \varepsilon$, and $n$. That is, there exist constants $C=$ $C(M, \varepsilon, n)$ and $c=c(M, \varepsilon, n)$ such that $C>c>0$ and the Bergman kernel for $P$ can be estimated as

$$
\begin{aligned}
& c \cdot \sum_{\mathcal{A} \in \mathbb{A}}\left(\prod_{j=1}^{k} \frac{1}{\left(1-\left|\left(z_{0} / z_{\mathcal{F}}\right)^{\alpha_{j}}\right|\right)^{2}} \cdot \sum_{\beta+\overline{\mathbf{1}} \in \mathcal{U}(\mathcal{A})} \frac{\left|z_{0}^{\beta}\right|^{2}}{\left\|\zeta_{\beta}\right\|^{2}}\right) \\
& \leq K_{P}\left(z_{0}, z_{0}\right) \\
& \quad \leq C \cdot \sum_{\mathcal{A} \in \mathbb{A}}\left(\prod_{j=1}^{k} \frac{1}{\left(1-\mid\left(z_{0} / z_{\mathcal{F}}\right)^{\left.\alpha_{j} \mid\right)^{2}}\right.} \cdot \sum_{\beta+\overline{\mathbf{1}} \in \mathcal{U}(\mathcal{A})} \frac{\left|z_{0}^{\beta}\right|^{2}}{\left\|\zeta_{\beta}\right\|^{2}}\right),
\end{aligned}
$$

where $\log |z \mathcal{F}| \in \mathcal{F}=\mathcal{F}(\mathcal{A})$.

REMARK 3.1. $\left|z_{\mathcal{F}}^{\alpha_{j}}\right|$ does not depend on the choice of $z_{\mathcal{F}}$ as long as $\log \left|z_{\mathcal{F}}\right| \in$ $\mathcal{F}(\mathcal{A})$ and $\alpha_{j} \in \mathcal{A}$. 


\section{References}

[1] J. Bruna, A. Nagel, and S. Wainger, Convex hypersurfaces and Fourier transforms, Ann. of Math. (2) 127 (1988), 333-365.

[2] D. Catlin, Boundary invariants of pseudoconvex domain, Ann. of Math. (2) 120 (1984), 529-586.

[3] - Estimates of invariant metrics on pseudoconvex domains of dimension two, Math. Z. 200 (1989), 429-466.

[4] D. C. Chang, A. Nagel, and E. M. Stein, Estimates for the $\bar{\partial}-$ Neumann problem in pseudoconvex domains of finite type in $\mathbb{C}^{2}$, Acta Math. 169 (1992), 153-228.

[5] J. D'Angelo, Real hypersurfaces, orders of contact, and applications, Ann. of Math. (2) 115 (1982), 615-637.

[6] L. Hörmander, An introduction to complex analysis in several variables, 3rd ed., North-Holland, Amsterdam, 1990.

[7] J. McNeal, Boundary behavior of the Bergman kernel function in $\mathbb{C}^{2}$, Duke Math. J. 58 (1989), 499-512.

[8] - Estimates on the Bergman kernels of convex domains, Adv. Math. 109 (1994), 108-139.

[9] A. Nagel, J. P. Rosay, E. M. Stein, and S. Wainger, Estimates for the Bergman and Szegö kernels in $\mathbb{C}^{2}$, Ann. of Math. (2) 129 (1989), 113-149.

Department of Computer Sciences

Purdue University

Lafayette, IN 47907-1398

tiaoch@cs.purdue.edu 